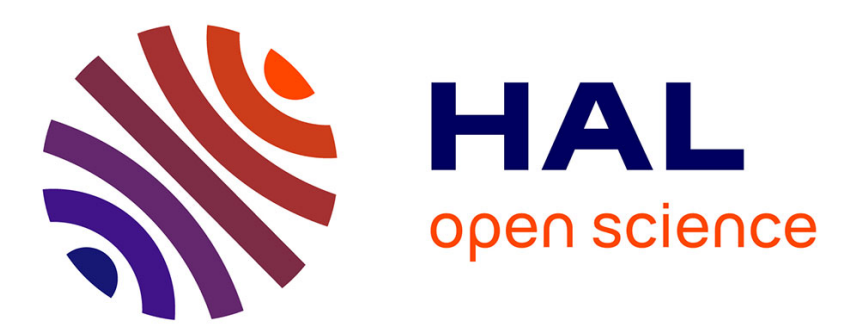

\title{
Etude de la topographie du travail de sortie du silicium et de l'oxyde de silicium
}

J.L. Chartier, L. Pilorget, R. Le Bihan

\section{To cite this version:}

J.L. Chartier, L. Pilorget, R. Le Bihan. Etude de la topographie du travail de sortie du silicium et de l'oxyde de silicium. Revue de Physique Appliquée, 1984, 19 (11), pp.927-931. 10.1051/rphysap:019840019011092700 . jpa-00245285

\section{HAL Id: jpa-00245285 https://hal.science/jpa-00245285}

Submitted on 1 Jan 1984

HAL is a multi-disciplinary open access archive for the deposit and dissemination of scientific research documents, whether they are published or not. The documents may come from teaching and research institutions in France or abroad, or from public or private research centers.
L'archive ouverte pluridisciplinaire HAL, est destinée au dépôt et à la diffusion de documents scientifiques de niveau recherche, publiés ou non, émanant des établissements d'enseignement et de recherche français ou étrangers, des laboratoires publics ou privés. 


\title{
Etude de la topographie du travail de sortie du silicium et de l'oxyde de silicium (*)
}

\author{
J. L. Chartier, L. Pilorget et R. Le Bihan \\ Laboratoire de Physique des Surfaces ( $\left.{ }^{*}\right)$, Institut de Physique, Université de Nantes, 44072 Nantes Cedex, France
}

(Reçu le 16 mai 1984, accepté le 31 juillet 1984)

\begin{abstract}
Résumé. - Dans le but d'étudier l'homogénéité et la qualité d'oxydes de silicium la topographie du travail de sortie a été déterminée sur des oxydes de différentes épaisseurs ( $80 \AA$ à $550 \AA$ ). L'influence de différents paramètres est présentée : l'épaisseur de l'oxyde, son processus de fabrication (ambiance sèche ou humide...) et les traitements subis (nettoyages...). Après avoir enlevé la couche d'oxyde les topographies du travail de sortie de substrats de silicium sont mesurées. Les différents échantillons sont analysés par spectrométrie Auger. L'influence du décapage ionique et du bombardement électronique sur la mesure du travail de sortie est présentée.
\end{abstract}

\begin{abstract}
In order to study the homogeneity and the quality of silicon oxides the work function topography has been measured on oxides of different thicknesses $(80 \AA$ to $550 \AA)$. The influence of different parameters is presented : oxide thickness, fabrication process (dry or wet atmosphere...) and the surface preparation before oxidation (oxygen sputter etch...). After removing the oxide film with a solution of fluorhydric acid the work function topography of the silicon substrate is measured. The samples are also studied by Auger electron spectroscopy. The influence of ion etching and electron beam on the work function measurement is presented.
\end{abstract}

\section{Introduction.}

Le développement de la microélectronique conduit à la réalisation de circuits à faible coût de plus en plus performants dans un volume le plus restreint possible. En particulier on est amené à réduire l'épaisseur des couches d'oxyde de silicium à moins de $100 \AA$, tout en conservant leur homogénéité, leur reproductibilité et en ayant les caractéristiques électriques désirées.

Nous nous sommes attachés à l'étude des couches minces d'oxyde de silicium, étude de leur qualité et en particulier de leur homogénéité quand on réduit leur épaisseur de $550 \AA$ à des valeurs d'environ 80 à $100 \AA$. Ces études sont conduites dans le cadre du GCIS et avec la contribution financière, scientifique et technique de Matra Harris Semiconducteurs (M.H.S.) qui nous a fourni les oxydes fabriqués de manière industrielle dans différentes conditions par B. Cambou et son équipe.

\section{Partie expérimentale.}

Les oxydes minces étudiés dont l'épaisseur varie de 80 à $550 \AA$, sont poussés thermiquement par différents processus et sur des substrats de silicium d'origines diverses mais tous d'orientation (100).

(*) U.A. CNRS No 788.
Le paramètre choisi pour l'étude de la qualité et de l'homogénéité des oxydes minces est le travail de sortie $\phi$ à cause de sa grande sensibilité aux propriétés de surface et parce qu'il peut être mesuré sans perturber l'échantillon étudié. La mesure de $\phi$ est effectuée par la méthode du condensateur vibrant ou sonde de Kelvin [1]. En déplaçant la sonde (de dimensions $0,4 \times 0,5 \mathrm{~mm}^{2}$ ) devant l'échantillon, nous réalisons une topographie du travail de sortie sur la surface de l'échantillon. Il s'agit de mesures relatives, un point de l'échantillon étant choisi comme référence pour toute la surface étudiée. Auparavant nous avons comparé les mesures du travail de sortie par la sonde de Kelvin à celles effectuées au microscope électronique à miroir dans la même enceinte à ultravide [2].

Les échantillons étudiés de $1 \times 2 \mathrm{~cm}^{2}$ environ sont découpés dans un wafer suivant deux directions perpendiculaires afin d'éviter des variations dues à un artefact. Les mesures ne sont pas effectuées sur le pourtour de l'échantillon de manière à éviter les effets de bord. Nous avons procédé à des études sous atmosphère ambiante et sous vide à une pression comprise entre $10^{-7}$ et $10^{-9}$ torr; dans les deux cas, les résultats obtenus étaient similaires. La préparation des échantillons peut se résumer en un nettoyage à l'alcool pour la plupart. Une cinquantaine d'échantillons ont ainsi été étudiés. 
Pour compléter leur caractérisation, les différents échantillons étudiés sous vide sont analysés par spectrométrie Auger à l'aide d'un dispositif C.M.A. placé dans la même enceinte à ultra-vide.

\section{Résultats. Discussion.}

3.1 ToPographie DU TRAVAIL DE SORTIE. - Les mesures réalisées sur tous les échantillons révèlent des variations du travail de sortie sur la surface d'oxyde. Les figures 1 à 3 montrent des topographies obtenues sur des oxydes d'épaisseur $110 \AA$, $250 \AA$ et $550 \AA$. Du point de vue travail de sortie, la surface n'est donc pas homogène. L'amplitude des variations diffère suivant les échantillons : elle est de l'ordre de $50 \mathrm{meV}$ pour une topographie " plate», pour atteindre 250 à $300 \mathrm{meV}$ sur une surface plus accidentée. Les

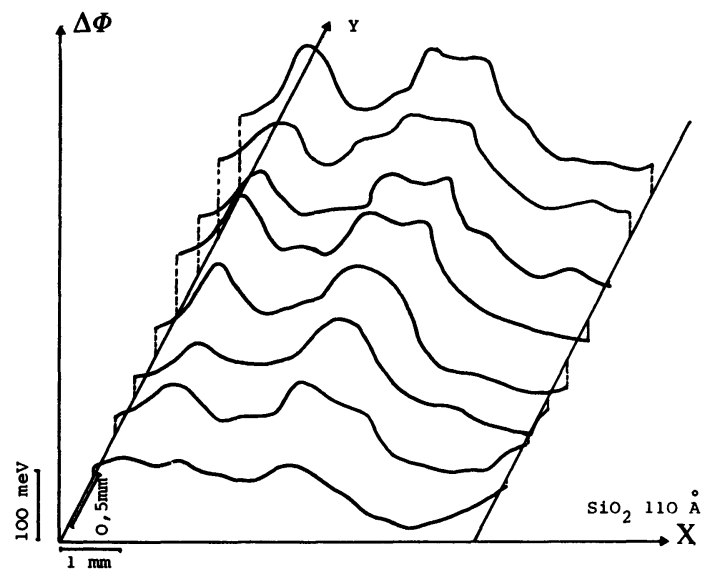

Fig. 1. - Topographie du travail de sortie d'un oxyde mince d'épaisseur $110 \AA$ relevée sous pression atmosphérique.

[Work function topography of silicon oxide of $110 \AA$ thickness obtained at room atmosphere.]

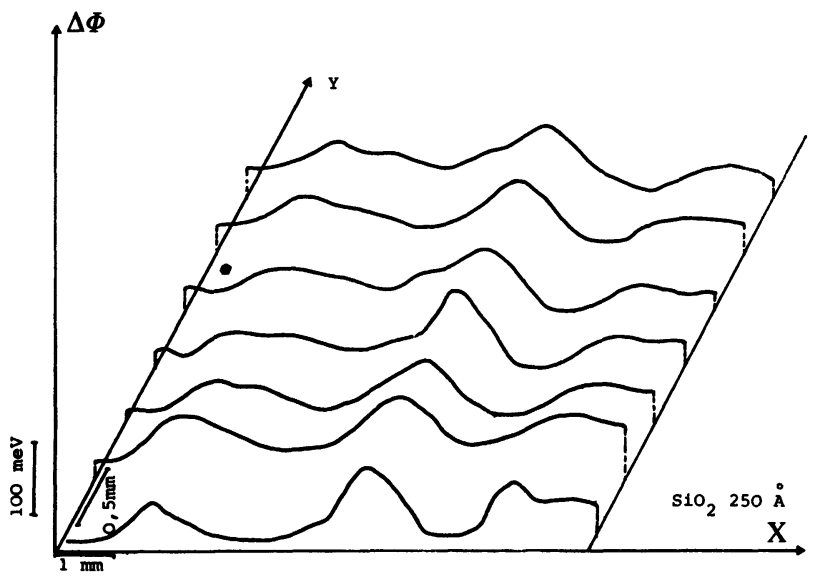

Fig. 2. - Topographie du travail de sortie d'un oxyde mince d'épaisseur $250 \AA$ relevée sous pression atmosphérique.

[Work function topography of silicon oxide of $250 \AA$ thickness obtained at room atmosphere.]

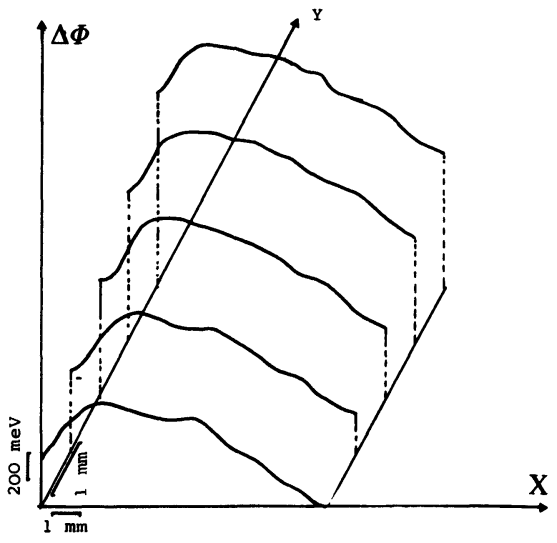

Fig. 3. - Topographie du travail de sortie relevée sous vide pour un oxyde d'épaisseur $550 \AA$.

[Work function topography of silicon oxide of $550 \AA$ thickness obtained under vacuum.]

variations de $\phi$ ont souvent la forme de sillons. Les mesures étant très sensibles à la distance sondeéchantillon, nous avons vérifié que ces variations du travail de sortie ne sont pas dues à des variations de la planéité de la surface des échantillons. Les mesures à l'alpha-step sur les mêmes surfaces montrent des courbures maximales de $0,1 \mu \mathrm{m}$ sur une distance de $3 \mathrm{~mm}$. Cette distance est négligeable car elle produirait une variation $\Delta \phi$ inférieure à la sensibilité du dispositif.

Nous avons étudié l'influence de différents paramètres sur la topographie. Dans la gamme d'épaisseur d'oxydes étudiées, nous n'avons pas trouvé de corrélation nette entre épaisseur d'oxyde et amplitude des variations du travail de sortie. Par contre, le processus de fabrication de l'oxyde (ambiance sèche ou humide...) et les traitements subis (nettoyages...) peuvent influencer la topographie du travail de sortie. Ainsi, un oxyde "wet " présente des variations moins importantes qu'un oxyde "Dry». De même lorsque le substrat a subi un décapage par plasma d'oxygène avant oxydation, l'oxyde présente une topographie du travail de sortie plus " plate ".

L'étude montre une grande sensibilité du travail de sortie à la luminosité; une modification de l'intensité lumineuse entraîne un décalage des courbes en valeur absolue mais ne modifie pas le profil d'où l'intérêt d'effectuer les mesures dans l'obscurité.

3.2 ETUDE Des SUbSTRATS DE SILICIUM. - L'étude des oxydes a été complétée par l'étude de leurs supports de silicium. La couche d'oxyde poussée sur le substrat est ôtée à l'aide d'une solution à base d'acide fluorhydrique avant introduction sous vide. Les variations du travail de sortie sur les échantillons de silicium (Fig. 4) se présentent sous la même forme que celles relevées sur les oxydes. L'amplitude des variations de $\phi$ qui se situe entre 150 et $200 \mathrm{meV}$ est du même ordre de grandeur que celles mesurées sur les oxydes. 


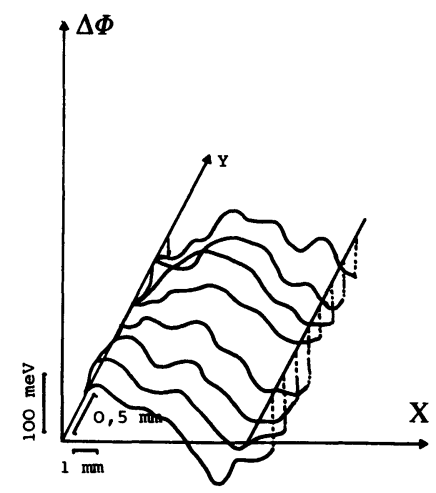

Fig. 4. - Topographie du travail de sortie d'un échantillon de silicium type $\mathrm{N}(100)$.

[Work function topography of silicon N (100) sample.]

3.3 INFLUENCE DE L'ÉPAISSEUR DE L'OXYDE SUR LE TRAVAIL DE SORTIE. - Comme nous l'avons vu ci-dessus, l'amplitude moyenne des variations du travail de sortie ne dépend pas de l'épaisseur des oxydes analysés. Pour comparer les valeurs absolues du travail de sortie des oxydes minces, nous avons collé sur le support trois échantillons d'oxydes d'épaisseurs différentes et un substrat de silicium de type N (100) dopé phosphore. Ce dernier a été décapé à l'acide fluorhydrique pour éliminer l'oxyde natif en surface. Les trois échantillons d'oxyde sont nettoyés à l'alcool, rincés à l'eau désionisée et séchés avant leur introduction sous ultra-vide pour la mesure du travail de sortie.

Les résultats des mesures (Fig. 5) montrent que les valeurs moyennes du travail de sortie sont sensiblement les mêmes pour les trois couches d'oxyde. La différence avec le travail de sortie du substrat de silicium est d'environ $200 \mathrm{meV}$.

3.4 INFLUENCE DU DÉCAPAGE IONIQUE ET DU BOMBARDEMENT ÉLECTRONIQUE SUR LA MESURE DU TRAVAIL DE SORTIE. - Pour ôter la couche adsorbée en surface

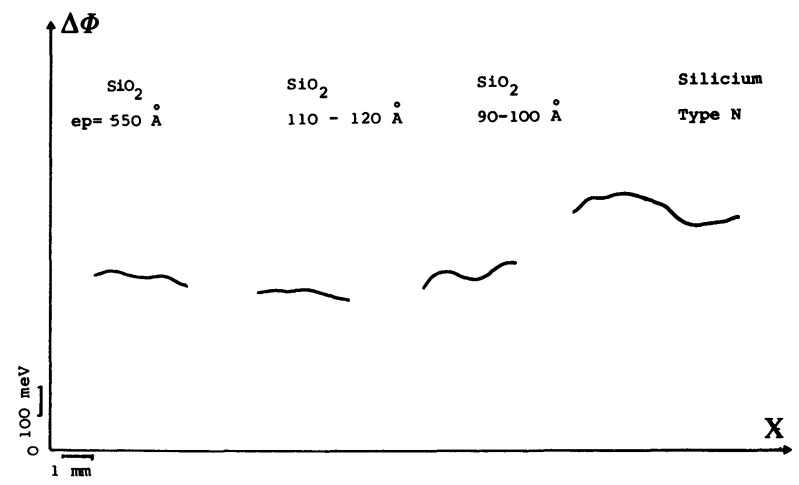

Fig. 5. - Topographie du travail de sortie de trois oxydes minces d'épaisseur différente et d'un silicium type $N$ (100) montés sur le même porte échantillon.

[Work function topography for three silicon oxide samples of differents thickness and for a silicon N (100) mounted on the same holder.] de l'oxyde, nous utilisons un décapage ionique. La surface de l'échantillon est bombardée par un faisceau d'ions argon qui balaie uniformément la surface. L'effet du nettoyage se traduit par une diminution d'amplitude des variations du travail de sortie.

L'influence d'un faisceau d'électrons sur une surface d'oxyde a déjà été étudiée par plusieurs auteurs [3, 4]. Elle met en évidence une nette perturbation de la surface au point d'impact du faisceau. Ici nous avons étudié l'influence d'un faisceau d'électrons qui a balayé la surface uniformément. Après un tel bombardement électronique, la topographie du travail de sortie est très aplatie ainsi que le montre la figure 6 .

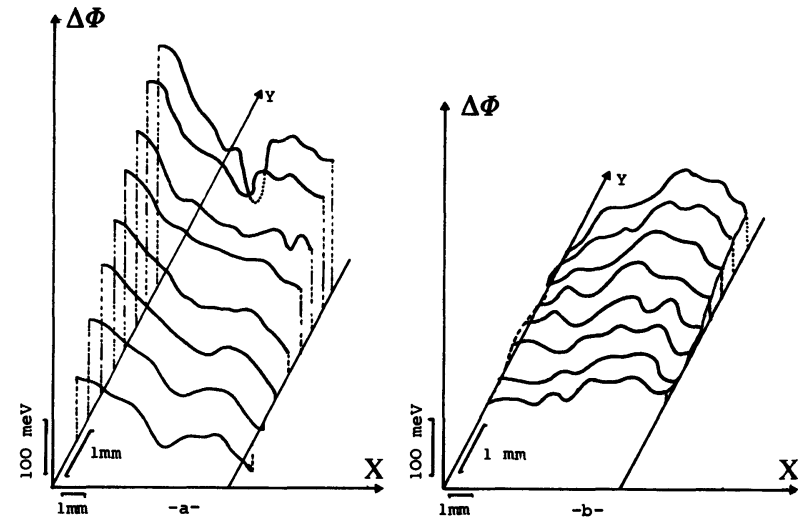

Fig. 6. - a) Topographie du travail de sortie d'un oxyde mince de silicium d'épaisseur $110 \AA$. b) Topographie du travail de sortie sur le même oxyde après bombardement électronique de la surface.

[a) Work function topography of a silicon oxide of $110 \AA$ thickness. b) Work function topography of the same oxide after electron beam bombardment.]

Les échantillons d'oxydes de différentes épaisseurs dont les mesures de topographie du travail de sortie ont été présentées figure 5 , ont été soumis à un balayage électronique. Les nouvelles topographies du travail de sortie relevées dans les mêmes conditions, sont présentées sur la figure 7. Nous observons une différence importante entre les oxydes d'épaisseur différente et le substrat de silicium. Cette différence est inversée et grandit avec l'épaisseur de l'oxyde pour atteindre environ $1000 \mathrm{meV}$ pour l'oxyde d'épaisseur $550 \AA$. Cette variation importante du travail de sortie induite par le bombardement électronique est à attribuer à la charge des oxydes en surface, la charge emmagasinée augmentant en fonction de l'épaisseur d'oxyde.

Nous avons également réalisé les expériences suivantes. On bombarde la surface de l'échantillon à l'aide du faisceau d'électrons maintenu fixe, en choisissant une densité du courant primaire constante et inférieure à $1,27 \times 10^{-2} \mathrm{~A} \mathrm{~cm}^{-2}$, densité susceptible de dissocier les molécules adsorbées sur la surface en fournissant un dépôt de carbone $[5,6]$ ou de provoquer la dissociation de l'oxyde de silicium [7]. Lors 


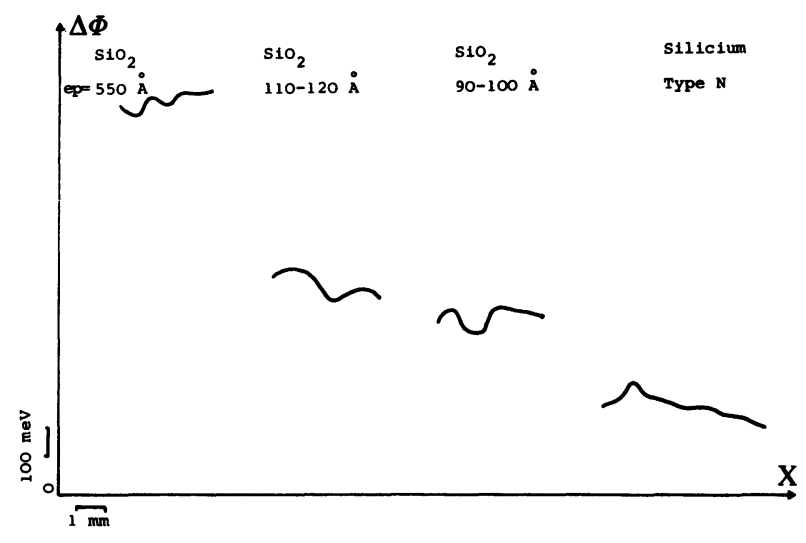

Fig. 7. - Topographie des échantillons de la figure 5 après bombardement électronique uniforme de leur surface.

[Work function topography of samples of figure 5 after bombardment by a scanning electron beam.]

de ce bombardement nous avons mesuré une variation du courant absorbé par l'échantillon tel que le montre la figure 8. Pour les échantillons d'oxyde, on obtient un courant qui décroît puis augmente légè-
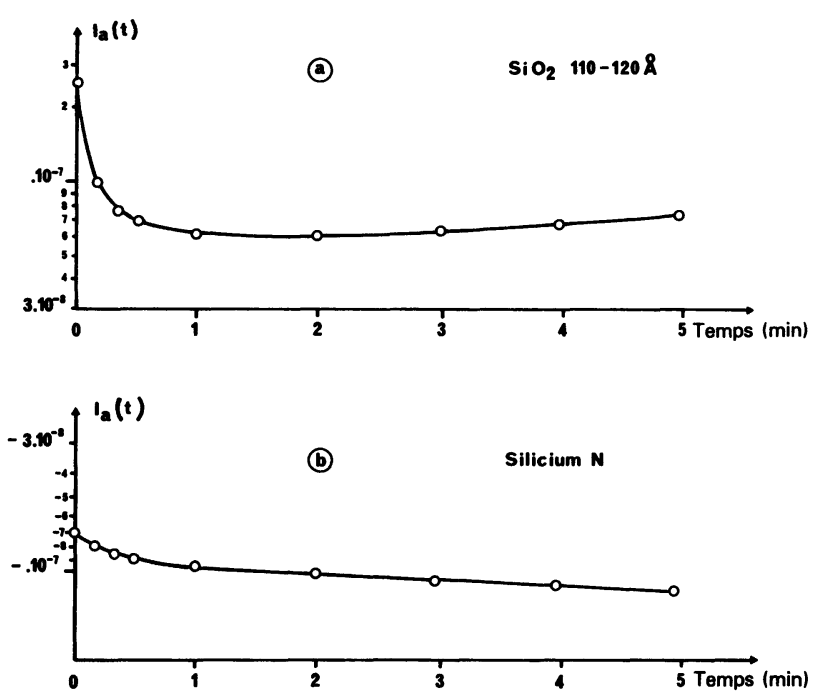

Fig. 8. - Evolution du courant absorbé par l'échantillon au cours du temps pour un courant incident constant : a) sur un oxyde mince d'épaisseur $110-120 \AA$; b) sur du silicium type $\mathrm{N}$ (100).

[Absorbed current variation during bombardment by a constant incident electron beam : a) for a $110 \AA$ thickness silicon oxide sample; b) for a silicon $\mathrm{N}$ (100) sample.]

rement avant de se stabiliser à une valeur très peu différente du minimum. Le courant absorbé par le silicium est de sens opposé, il varie moins et se stabilise à sa valeur minimale. Ces variations du courant absorbé sont dues à la charge des échantillons sous l'effet du bombardement électronique comme ceci a été étudié par ailleurs [8].
3.5 Etude Par SPECTROMÉtrie Auger. - Les différents échantillons étudiés ci-dessus sous vide ont été analysés par spectrométrie Auger. Voyons la comparaison d'un oxyde obtenu par croissance sous « $\mathrm{O}_{2}$ wet " d'épaisseur 110-120 $\AA$ avec un autre obtenu sous " $\mathrm{O}_{2}$ dry " et ayant une épaisseur de 90-100 ̊. Les spectres relevés avant nettoyage de la surface font apparaître le pic du silicium oxydé décalé en énergie et la présence de carbone à la surface. Après un léger décapage ionique aux ions argon, les spectres révèlent une surface propre, le pic du carbone a disparu et le pic du silicium oxydé se trouve à sa valeur nominale de $76 \mathrm{eV}$, l'oxygène se trouvant à l'énergie de $507 \mathrm{eV}$ (Fig. 9a et b). La spectrométrie Auger ne fait pas apparaître de différence de composition de surface entre les deux oxydes analysés. Il en est de même pour la comparaison avec les spectres obtenus pour des couches d'oxydes plus épaisses (Fig. 9c). L'énergie des liaisons en surface est la même sur tous les échantillons analysés.

Lors de l'étude du substrat de silicium, nous avons constaté que la disparition de toutes traces de conta-

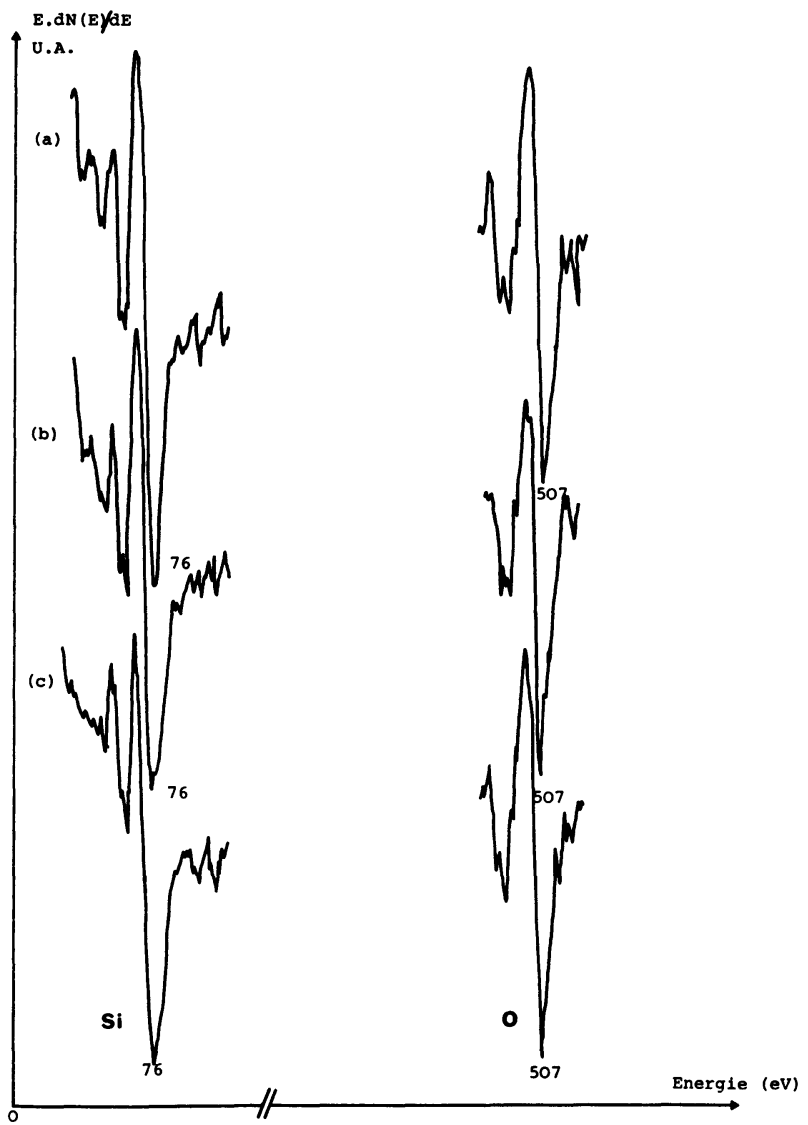

Fig. 9. - Spectres Auger dérivés relevés sur des oxydes de silicium : a) oxyde mince d'épaisseur $90-100 \AA$ - croissance sous « $\mathrm{O}_{2}$ wet "; b) oxyde mince d'épaisseur $110-120 \AA-$ croissance sous " $\mathrm{O}_{2}$ dry »; c) oxyde mince d'épaisseur $550 \AA$

[Auger spectra of silicon oxides : a) $90-100 \AA$ oxide thickness - grow under $\mathrm{O}_{2}$ wet; b) $110-120 \AA$ oxide thickness - grow under $\mathrm{O}_{2}$ dry; c) $550 \AA$ oxide thickness.] 
minants à la surface du silicium, en spectrométrie Auger, nécessite le chauffage de l'échantillon sous ultra-vide.

\section{Conclusion.}

L'étude réalisée a permis de mettre en évidence des variations du travail de sortie à la surface de l'oxyde de silicium. Pour les couches d'oxyde étudiées dont les épaisseurs variaient de $80 \AA$ à $550 \AA$, les paramètres de la fabrication et les traitements avant oxydation se sont révélés plus influents que l'épaisseur de l'oxyde sur la topographie du travail de sortie. Ainsi on a vu qu'un traitement sous plasma d'oxygène avant oxydation fournit une topographie plus plate pour le travail de sortie. La variation du travail de sortie se retrouve sur le substrat de silicium. D'où l'intérêt de l'étude du début de l'oxydation du silicium à laquelle nous nous sommes intéressés par ailleurs. Cette recherche devrait contribuer à déterminer l'explication de ces variations du travail de sortie. Après un léger nettoyage ionique de la surface, l'analyse Auger ne fait pas apparaître de différence de composition de surface entre les oxydes dans la gamme des épaisseurs étudiées $(80$ à $550 \AA)$.

Il est important maintenant de voir s'il y a une corrélation entre les variations de la topographie du travail de sortie et les propriétés électriques des dispositifs électroniques réalisés avec ces oxydes. Les mesures du travail de sortie doivent être réalisées dans les mêmes conditions d'atmosphère que celles existant avant le processus suivant l'oxydation.

\section{Bibliographie}

[1] Zissman, W., Rev. Sci. Instrum. 3 (1932) 367.

[2] Le Bihan, R., Chartier, J. L., Entretiens de Toulouse sur la microélectronique (GCIS), 1982.

[3] Allen, E. G., Phys. Chem. Solids 8 (1959) 119.

[4] Testemale, E., Palau, J. M., Poulvil, P., Lassabatère, L., Le Goascoz, V., Thin Solid Films 71 (1980) 161-176.
[5] CoAd, J. P., Bishop, D. H., Rivière, J. C., Surface Sci. 21 (1970) 253.

[6] Joyce, B. A., Neave, J. H., Surface Sci. 34 (1973) 401.

[7] Carrière, B., LaNG, B., Surface Sci. 64 (1977) 209-223.

[8] Le Bihan, R., Le Vide, Les Couches minces 36 (206) (1981) 367-381.

(*) Communication présentée aux Journées du G.C.I.S., Toulouse le 15 et 16 décembre 1983. 